\title{
EXPERIENCIAS DOCENTES APOYADAS EN AULAS VIRTUALES
}

\author{
(EDUCATIONAL EXPERIENCES SUPPORTED IN VIRTUAL CLASSROOMS)
}

Francisco Pavón Rabasco

Juan Casanova Correa

Universidad de Cádiz (España)

\section{RESUMEN}

En este artículo pretendemos exponer parte de nuestra experiencia docente de los últimos años apoyada en campus virtuales y además de relacionar las muchas ventajas que nos han proporcionado, también los posibles inconvenientes o necesidades de adaptación tanto del profesorado como de los estudiantes a la nueva herramienta.

Palabras clave: enseñanza virtual, innovación docente virtual, e-learning universitaria.

\begin{abstract}
In this article we try to expose part of our educational experience of the last years supported in virtual campus and besides to relate the many advantages that have provided us, also the possible disadvantages or adaptation' necessities for teachers as students using the new tool.
\end{abstract}

Key words: Virtual teaching, virtual educational innovation, university e-learning.

\section{EL APOYO VIRTUAL COMO RECURSO DIDÁCTICO EN LA ENSEÑANZA UNIVERSITARIA}

Como profesores de Universidad, nos estamos preparando para adaptar la impartición de nuestras asignaturas a las orientaciones que nos dan de cara al EEES. Una de ellas surge a partir de la Cumbre de Lisboa de 2000, donde se puso en marcha el plan e-Europe, que tiene como objetivos formativos "adaptar los curricula, 
para hacer posible nuevas formas de aprendizaje, utilizando las Tecnologías de la Información y de la Comunicación”.

También desde nuestra Universidad se nos informa que el uso de las Nuevas Tecnologías y la enseñanza virtual es uno de los grandes retos a abordar dentro del proceso de convergencia, en el que se combina el trabajo presencial en el aula o en el laboratorio con la enseñanza a través de Internet y que permitirán minimizar las limitaciones de espacio y tiempo que exige la enseñanza convencional, flexibilizando los procesos de aprendizaje y aprovechando al máximo los recursos de las tecnologías digitales.

Según Sancho (2003), en una época en la que todo el mundo parece esperar mucho de la educación, una aportación fundamental de las teorías del aprendizaje es que diferentes tipos de finalidades de aprendizaje requieren diferentes aproximaciones a la enseñanza. Lo que lleva a plantear que los entornos de aprendizaje tradicionales centrados en el docente se han revelado como insuficientes para propiciar las finalidades educativas de la mayoría de los sistemas educativos contemporáneos. De este modo, dar respuesta a las necesidades educativas de la sociedad actual implica cambiar la imagen de una enseñanza fundamentalmente centrada en el profesorado, para dibujar entornos de enseñanza diversificados en que se comience a considerar el papel del alumnado, el conocimiento, la evaluación y la comunidad.

Los entornos centrados en el alumnado se asemejan bastante a los que están surgiendo en la mayoría de las universidades en sus campus virtuales, donde se requiere un profesorado, con actitudes y aptitudes diferentes, consciente de que los estudiantes construyen sus propios significados, comenzando con las creencias, las comprensiones y las prácticas culturales que traen. Si la enseñanza se entiende como la construcción de un puente entre las materias de estudio y los estudiantes, los docentes que se centran en el alumnado no pierden de vista ninguna de las dos partes del puente. El profesorado intenta entender lo que el alumnado sabe y puede hacer además de sus intereses y pasiones: qué conoce cada estudiante, le interesa, sabe y quiere hacer.

El aula virtual es un entorno educativo que intenta facilitar el aprendizaje cooperativo y colaborativo entre estudiantes y entre éstos y los profesores. Las técnicas didácticas usadas en educación con redes, se pueden clasificar en función del número de emisores/receptores de la comunicación: 
- $\quad$ Técnicas "uno-solo". Se trata normalmente de aplicaciones cliente/servidor. Están basadas en el paradigma de la recuperación de la información de Internet.

- Técnicas "uno-a-uno". La comunicación se establece básicamente entre dos personas. Es por tanto individual y personalizada, basada en el texto y sometida a las características del medio.

- Técnicas "uno-a-muchos". Están basadas en aplicaciones como el correo electrónico que permiten la comunicación entre el profesor y un grupo de alumnos numeroso.

- Técnicas "muchos-a-muchos". Todos tienen oportunidad de participar en la interacción y todos pueden ver las aportaciones de los demás, profesores, estudiantes, expertos invitados, etc.

Pero no se puede perder de vista que los entornos solamente centrados en el alumnado no siempre ayudan a adquirir el conocimiento y las habilidades que se necesitan para vivir en sociedad.

De ahí que necesitemos crear entornos centrados en el conocimiento donde se plantee que los estudiantes sean personas cultas y que aprendan de un modo que les facilite la comprensión y la transferencia. Los entornos centrados en el conocimiento se entrecruzan con los que tienen en cuenta al alumnado cuando la enseñanza comienza con el interés no sólo por los preconceptos del estudiante sobre los temas de estudio, sino también por su forma de aprender.

Trataremos de justificar y dar sentido a las lecturas y tareas que les sugeriremos a lo largo del curso, para ayudar al alumnado a desarrollar procesos de transferencia hacia el aula de cada cual.

Los intentos de crear entornos centrados en el conocimiento también plantean cuestiones importantes sobre cómo favorecer la comprensión integrada de una disciplina o sobre cómo abordar temas transdisciplinares. Muchos modelos de diseño de curriculum tienden a considerar el conocimiento y las habilidades más como elementos desconectados que como conjuntos interconectados. Es por eso que la mayoría de los curricula fracasan a menudo a la hora de ayudar a los estudiantes a seguir su camino en una disciplina o en un tema integrador de conocimientos relevantes. Un desafío para diseñar entornos centrados en el conocimiento es conseguir el equilibrio apropiado entre las actividades diseñadas 
para promover la comprensión y las diseñadas para promover la automaticidad de las habilidades necesarias para funcionar de forma efectiva sin sentirse desbordado por requerimientos que demandan atención.

Pero quizás, el mayor desafío para la planificación de los entornos basados en el conocimiento sea trascender la idea de que existe un orden psicológico guiado por el constructivismo (hay que adecuar lo que se enseña al nivel de desarrollo del alumnado) y un orden disciplinar vinculado a una selección de materias y contenidos que hay que secuenciar de manera ordenada y estable en un proyecto curricular de escuela, de ciclo, de aula.

Los entornos de enseñanza que intentan responder a las necesidades del alumnado, además de centrarse en el aprendiz y el conocimiento, han de estar centrados en la evaluación. Los principios clave de la evaluación son proporcionar oportunidades para la realimentación y la revisión. Mientras que lo que se evalúa ha de ser coherente con las metas educativas, los procesos y los recursos.

Un desafío para poner en acción prácticas adecuadas de evaluación implica cambiar los modelos de muchos docentes, estudiantes, personal de apoyo y de la Administración sobre lo que significa un aprendizaje "efectivo". Muchas evaluaciones desarrolladas por docentes ponen el énfasis en la memorización de procedimientos y hechos. Sin embargo, las evaluaciones diseñadas de forma apropiada pueden ayudar al profesorado a darse cuenta de la necesidad de repensar sus prácticas de enseñanza.

Pero sobre todo, el sistema de evaluación ha de ser coherente con las finalidades educativas, estar presente en todo el proceso de enseñanza y aprendizaje y utilizar recursos diversificados para que el alumnado pueda mostrar realmente lo que sabe de la forma que le resulte más adecuada a su manera personal de aprender. De este modo, aunque parece casi imposible sustraerse de la dimensión "punitiva" y acreditativa de la evaluación, profesorado y alumnado pueden encontrar en este proceso un marco de diálogo desde el que calibrar sus mutuos avances.

Finalmente, las teorías contemporáneas del aprendizaje sugieren que en la medida en que los entornos de enseñanza estén centrados en la comunidad, se favorece las condiciones de aprendizaje para la mayoría del alumnado. De aquí que sea especialmente importante la creación de situaciones en las que unos puedan aprender de otros y lleven a una mejora continuada de la enseñanza. Como ejemplo, todos tendremos experiencias personales en las que hemos aprendido a resolver 
un problema de ordenador gracias a los consejos recibidos por algún compañero o compañera, no necesariamente informático.

\section{LA FORMACIÓN DEL PROFESORADO UNIVERSITARIO EN ESTAS HERRAMIENTAS: LOS CURSOS, LOS GRUPOS DE FORMACIÓN Y LAS INICIATIVAS PARA LA INNOVACIÓN EDUCATIVA}

El modelo que utilizamos en nuestra Universidad para actualizarnos y perfeccionarnos en nuevas metodologías docentes, especialmente ahora que estamos en puertas del Espacio Europeo de Educación Superior (EEES) es formar voluntariamente grupos y sus miembros trabajar colaborativamente en esa tarea.

El origen de la creación de los Grupos de Formación del Profesorado en la Universidad de Cádiz formó parte de un programa de la Unidad de Calidad de las Universidades Andaluzas (UCUA): el Plan Andaluz de Formación del Profesorado Universitario (PAFPU). En el curso 2005/2006, en el que la UCUA deja de tener competencias en materia de formación del profesorado, la Universidad de Cádiz, adoptó un programa propio, coordinado directamente desde el Vicerrectorado de Ordenación Académica e Innovación Educativa. No obstante, a estos Grupos de Formación hay que situarlos en el contexto más amplio del Plan de Acciones Formativas del Profesorado de la UCA, aprobado en su día en Consejo de Gobierno (30 de octubre de 2003).

A lo largo de estos años, se han planificado y llevado a cabo diversas acciones formativas, siendo las propuestas supervisadas y aprobadas por un equipo de formación. Esta comisión, además, tiene la misión de asesorar al Vicerrectorado de Ordenación Académica en la confección y planificación de las acciones formativas que se presentan como oferta abierta al conjunto del profesorado y colaborar para dar coherencia y dotar de coordinación al programa.

Cada uno de los miembros de esta comisión se responsabiliza de la planificación de actividades en el marco de las diferentes áreas temáticas o focos de interés formativo expresados por el profesorado de la Universidad de Cádiz: uso de nuevas tecnologías en el ámbito docente, la metodología docente, los contenidos de los nuevos planes de estudio, la evaluación. 


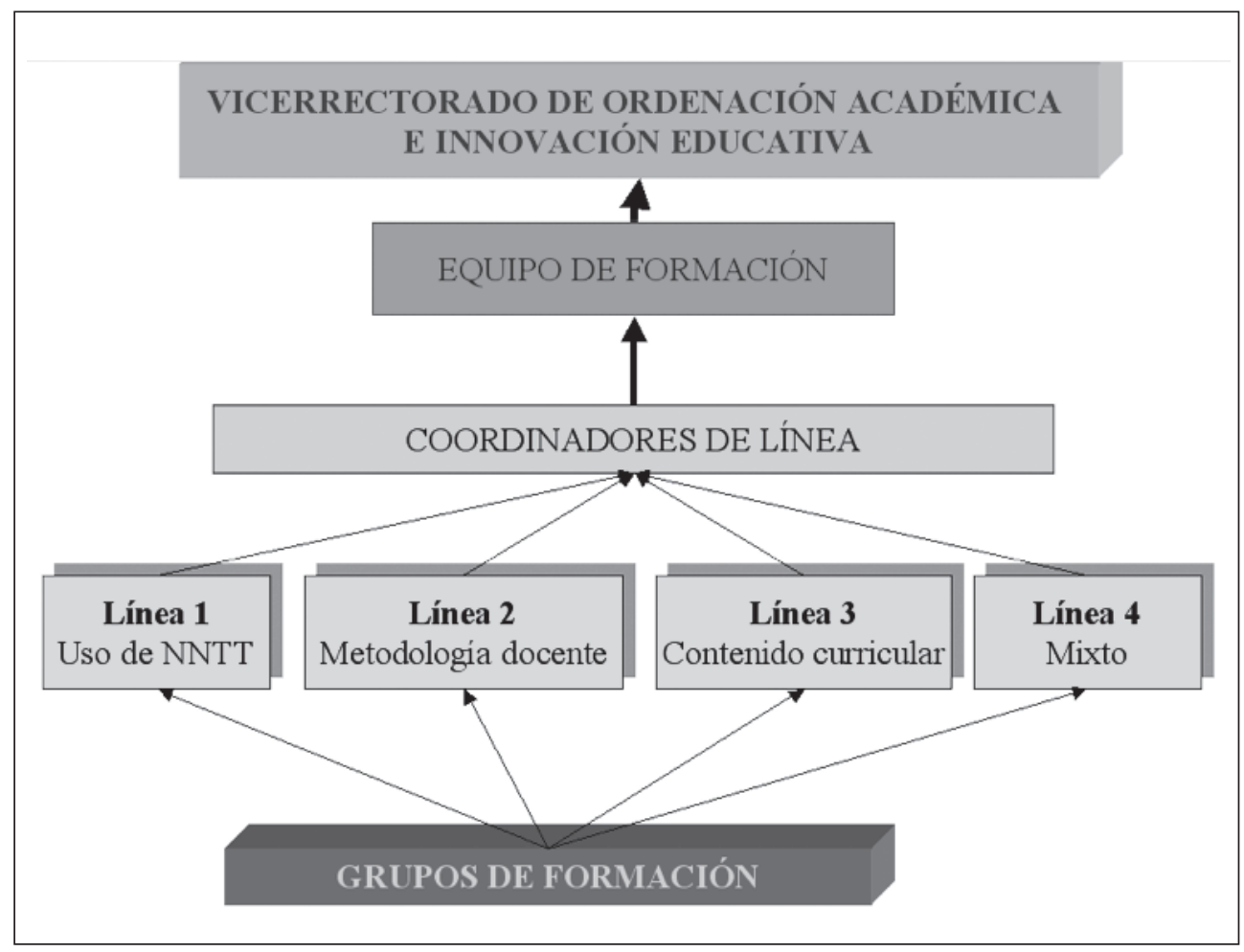

Figura 1: Organización de la formación del profesorado en la UCA

Cada uno de los responsables de estas áreas son coordinadores de alguno de los grupos de formación del profesorado, y representan al conjunto de grupos, o asociación, cuyo trabajo formativo se encuentra relacionado con el área temática de la que son responsables. Este sistema permite establecer canales de comunicación permanente entre los profesores y el Vicerrectorado en relación a sus inquietudes y necesidades formativas. El intercambio de experiencias y la relación entre las distintas asociaciones se consigue gracias a la Jornada de los Grupos de Formación que se celebra a principios del mes de julio.

En la figura 1 hemos intentado reflejar la idea organizativa que se desarrolla en nuestra Universidad respecto a la formación del profesorado. Pero, ¿qué criterios se siguen en el Plan de Acciones Formativas de la UCA? Seis son los criterios que se identifican: 
- La participación en el plan tiene un carácter voluntario.

- Nace con la intención de tratar de conciliar la demanda de formación con la oferta, para lo cual se realizará una consulta y análisis periódico sobre necesidades formativas.

- Tiene por objetivo promover la introducción de métodos participativos de enseñanza en la UCA, y debe contar como premisa con la implicación activa de los profesores.

- Las acciones deben contar con materiales de soporte previamente revisados para constatar su ajuste con los objetivos previstos.

- Los participantes que alcancen los objetivos establecidos para cada acción recibirán una certificación en la que conste la realización de la actividad.

- Las acciones formativas y el propio plan deben estar sometidas a controles periódicos de evaluación.

Si tuviésemos que definir en pocas palabras los criterios seleccionados, éstas serían: voluntariedad, adecuación y evaluación. Las ideas que sustentan este modelo están basadas en la participación directa y motivada de los auténticos agentes de la innovación en la Universidad: los docentes. De esta forma, la oferta formativa es demandada por los propios participantes y cumple el requisito de aplicación a la práctica docente cotidiana, ya que es el propio profesorado quien genera el conocimiento y la praxis en su quehacer docente. Por otro lado, se establecen mecanismos de evaluación que permiten recoger información sobre el proceso seguido, pero sobre todo, se recoge información que permite la autoevaluación de lo desarrollado a lo largo de la acción formativa del grupo de trabajo.

En fin, el plan pretende que aquellos que están interesados en introducir elementos innovadores en sus aulas (siguiendo la filosofía del Espacio Europeo de Educación Superior), puedan contar con un respaldo institucional (tanto económico como de medios), se les reconozca como actividades relevantes (reconocimiento de créditos tipo B -actividades no docentes-), y se les acredite a través de certificados de participación que son de especial interés para aquellos que todavía no tienen un contrato laboral estable.

Los grandes bloques que el plan pretende reforzar se concretan en los siguientes tipos de acciones formativas: 


\section{TIPOS DE ACCIONES FORMATIVAS EN LA UNIVERSIDAD DE CÁDIZ}

1.- Introducción a las tecnologías de la información y usos didácticos de las TIC's

2.- Seminarios formativos sobre la práctica docente

3.- Proyecto Andaluz de Formación del Profesorado Universitario

4.- Nuevas estructuras del sistema Universitario y sus procedimientos de gestión

5.- Acciones especiales de formación docente del profesorado

6.- Conocimiento de lenguas y culturas extranjeras para el impulso a iniciativas institucionales

7.- Seguridad y salud laboral

Figura 2: Tipos de acciones formativas ofrecidas desde la UCA

De los siete tipos recogidos en la figura 2, queremos resaltar dos de ellos: el primero y el quinto. El primero por el peso que las TIC's están teniendo en el proceso de adaptación de una Universidad, tanto al ámbito social como al ámbito educativoformativo, donde la presencia de tecnologías de transmisión de información es notable y cada vez más imprescindibles. Y el quinto, por lo que nos atañe como profesores de la facultad de Ciencias de a Educación en la que se imparte una titulación de segundo ciclo (Psicopedagogía) de carácter semipresencial, y que tiene como soporte al campus virtual de la UCA. Estos dos tipos giran en torno a las aportaciones de las TIC's en el buen hacer docente, o al menos, en intentar mantener un nivel competitivo ante otras universidades del entorno que las utilizan y que maximizan su uso con la captación de estudiantes que valoran su uso en los procesos de enseñanza-aprendizaje en el ámbito universitario. 


\section{EJEMPLOS DE USO DE HERRAMIENTAS VIRTUALES EN ASIGNATURAS DE LA FACULTAD DE CIENCIAS DE LA EDUCACIÓN DE LA UNIVERSIDAD DE CÁDIZ}

En la enseñanza reglada, nuestros estudiantes sólo utilizan la plataforma como apoyo a lo que venimos realizando en clase, pero termina convirtiéndose en una herramienta muy potente. En nuestro modelo de formación con apoyo de la plataforma, se trata de crear Comunidad Virtual de Aprendizaje, donde se aprenda con los demás, con una mayor implicación del alumno en actividades del grupo y con un incremento de la autonomía y la responsabilidad del estudiante respecto al propio proceso de aprendizaje.

Es una de las grandes ventajas de los entornos electrónicos donde las actividades grupales ofrecen un inmenso potencial. La premisa para la comunidad virtual de aprendizaje es efectivamente la colaboración (creación compartida). El tema no es la comunicación o el trabajo en equipo, sino la creación de valor. Se trata, en todo caso, de procesos de aprendizaje colaborativo, de lograr un contexto que enfatiza las interacciones inter e intragrupo donde los miembros participan autónomamente en un proceso de aprendizaje, mientras resuelven un problema como grupo (Salinas, 2003).

¿Cómo son estos procesos a los que tratamos de adecuarnos? Se trata como describe Prendes (2003b, p. 96) de "alumnos trabajando en grupo que intercambian ideas, se hacen preguntas, todos escuchan y comprenden las respuestas, se ayudan entre ellos antes de pedir ayuda al profesor y finalmente obtienen un único producto del trabajo del grupo" y de construcción del conocimiento.

Las comunidades de aprendizaje son esencialmente "comunidades de indagadores". Por lo tanto, los roles de "experto" y "alumno" se convierten en arbitrarios. Ambos son invitados al reino de la curiosidad conjunta.

Entonces, se debe entender que las actividades académicas desarrolladas en este campus virtual requieren aplicar los nuevos enfoques más centrados en el alumno y apoyados en la interacción, para poder ser considerados comunidades virtuales de aprendizaje. Es decir, se exigirá una interacción bastante amplia y una implicación y responsabilidad en el proyecto.

El modelo didáctico de las comunidades virtuales de aprendizaje es el aprendizaje colaborativo, que se centra en el alumno mejor que en el profesor y donde el 
conocimiento es concebido como un constructo social, facilitado por la interacción, la evaluación y la cooperación entre iguales. Según Prendes (2003a, p. 127) estamos ante un "método docente planificado, guiado, seguido y evaluado por el profesor que además contribuye al desarrollo intelectual, social y personal de los alumnos, enseñándoles a aprender y a cooperar".

Por consiguiente el rol del profesor cambia de la transmisión del conocimiento a los alumnos a ser facilitador en la construcción del propio conocimiento por parte de estos. Se trata de una visión de la enseñanza en la que el alumno es el centro o foco de atención y en el que el profesor juega, paradójicamente, un papel decisivo. Adoptar un enfoque de enseñanza centrada en el alumno significa atender cuidadosamente a aquellas actitudes, políticas y prácticas que pueden ampliar o disminuir la "distancia" de los alumnos distantes. El profesor actúa primero como persona y después como experto en contenido. Promueve en el alumno el crecimiento personal y enfatiza la facilitación del aprendizaje antes que la transmisión de información.

Los requerimientos a los profesores en este ámbito son:

- Guiar a los alumnos en el uso de las bases de información y conocimiento así como proporcionar acceso a los mismos para usar sus propios recursos.

- Potenciar alumnos activos en el proceso de aprendizaje autodirigido, en el marco de acciones de aprendizaje abierto, explotando las posibilidades comunicativas de las redes como sistemas de acceso a recursos de aprendizaje.

- Asesorar y gestionar el ambiente de aprendizaje en el que los alumnos están utilizando estos recursos. Tienen que ser capaces de guiar a los alumnos en el desarrollo de experiencias colaborativas, monitorizar el progreso del estudiante; proporcionar feedback de apoyo al trabajo del estudiante; y ofrecer oportunidades reales para la difusión de su trabajo.

- Acceso fluido al trabajo del estudiante en consistencia con la filosofía de las estrategias de aprendizaje empleadas y con el nuevo alumno-usuario de la formación descrito.

\section{¿Cómo será la acción tutorial en una comunidad virtual?}

A este respecto, Pérez i Garcías (2002) proporciona algunas recomendaciones para llevar a cabo la acción tutorial en una comunidad virtual: 
- Los alumnos necesitan adquirir habilidades técnicas y comunicativas. $\mathrm{Al}$ inicio del programa de formación es importante diseñar un conjunto de actividades para que el alumno adquiera habilidades con el nuevo entorno, tanto en aspectos técnicos como comunicativos.

- Asegurar una percepción adecuada de la actividad. Los grupos deben sentirse como grupos y desarrollar, normas y formas de entender comunes, como mecanismo de reducción de la ambigüedad.

- Disponibilidad tecnológica. Un adecuado acceso al sistema de comunicación durante el tiempo necesario para la comunicación.

- Establecer espacios de comunicación regulares públicos o privados para el feedback a las tareas y al progreso del alumno. El alumno, aún trabajando en grupo no debe sentirse aislado.

- Separar los espacios de comunicación de contenido con los de proceso.

Además de los espacios de comunicación propios de cada actividad es importante disponer de otros espacios de comunicación relacionados con el proceso de trabajo y la evolución del curso.

- Evitar la centralización del tutor en los procesos de comunicación. El docente debe ubicarse más como miembro del grupo que como experto.

- Apoyarse en los compañeros. Es interesante que el docente comparta el rol de moderador con alumnos que se responsabilizan de coordinar actividades o de proponer preguntas o respuestas a los compañeros.

- Dinamizar el trabajo del grupo. El tutor debe guiar y dinamizar el trabajo del grupo.

\section{VALORACIÓN DE LA EXPERIENCIA}

Dentro de este apartado vamos a tratar de dialogar o reflexionar en voz alta con las expresiones textuales manifestadas por nuestros estudiantes en la evaluación de los cursos on line.

- Se valora la virtualidad. En general, muchos coinciden en declarar que les hubiera sido imposible realizar el curso en una modalidad presencial debido a sus obligaciones profesionales y familiares: 
"El curso ha sido muy intenso, tiene todas las ventajas de un curso on-line y estar hablando de comunicación, Internet, educación y contenidos desde el pc de casa siempre te resulta más cómodo y rápido que asistir a cualquier sala. Además la libertad de horarios es una maravilla, como diría aquel, ésto no tiene precio".

"Para mi situación personal, realizar un curso presencial me resulta casi imposible".

- Se le ha perdido el miedo a las TIC's. Han cambiado de idea inicial de respeto a las TIC's y comprobado que el que se atreve con ellas, acaba sintiéndose seguro utilizándolas:

"El curso me ha parecido muy bueno, me parece estupendo la formación de los enseñantes en el campo de las nuevas tecnologías (las grandes desconocidas). Hemos podido observar que no es tan difícil como creemos, sólo es cuestión de enfrentarse a ellas y ver que las podemos manejar a la perfección".

"No exagero al decir que de todos los cursos que he hecho, éste ha sido el de más utilidad. Desde el lado personal, me siento más segura manejando el ordenador, navegando por Internet, he aprendido a hacer cosas nuevas..., en fin ya no me siento tan "torpe" con la informática, y esto me anima a seguir. Del lado profesional, he descubierto multitud de herramientas para utilizar en mis clases, que mis alumnos agradecen".

- Consideran la temática de gran interés. Básica para ser buen docente en el siglo XXI:

"Los cursos a través de la web son ideales para mi forma de trabajo. El de nuevas tecnologías me ha aportado una amplia visión de estos medios abriendo nuevos horizontes para mi labor como docente".

“...estos cursos sobre las NTIC no sólo son necesarios, yo aun podría añadir que, en la actualidad, aquellas personas que todavía no saben utilizar herramientas como Internet, el correo electrónico, etc. cada vez se quedan mas aisladas de la revolución que nuestra realidad esta sufriendo, y eso como ciudadanos nos aisla de la sociedad, pero como docentes, nos incapacita para desarrollar nuestra labor en un mundo en el que los conocimientos ya no residen en las personas,nosotros l@s profesor@s ya no somos el pozo de sabiduría del que beben nuestros alumnos..." 
"Estamos todos de acuerdo en la necesidad de acercar las nuevas tecnologías a la escuela y este curso ha servido para mostrarnos cómo, para y por qué hacerlo".

- Se comprende la estructura y se agradece la compañía. Han comprobado que lo virtual tiene también su calendario y no necesariamente implica soledad:

“...me ha encantado el calendario, la planificación y la distribución del curso, donde cada semana estaba muy claro lo que había que hacer".

"Me he sentido muy acompañado y centrado (ritmo claro y muy marcado de aprendizaje)".

- $\quad$ Se sale del curso con proyectos. Terminan con buenas propuestas, unos ya lo han puesto en práctica y otros guardan su deseo para el próximo curso:

"Lo mejor, aprender el manejo de utilidades multimedia/informáticas para poder emplear en clase. Mis alumnos han sabido valorar mi esfuerzo y eso se agradece".

“...me siento reconfortado con el curso. Ahora sólo me queda ponerlo en práctica, al menos ahora ya tengo algunos conocimientos para ello”.

"...es un gusto ver que hay cursos enriquecedores para la labor docente, y este ha sido uno de ellos. Creo que a partir de ahora muchos de nosotros cambiaremos el chip y nos esforzaremos por incorporar las nuevas tecnologías a nuestra ardua tarea de educar chicos y chicas, ciudadanos del futuro, y qué mejor que hacerlo con la herramienta del futuro".

- $\quad Y$ con intención de repetir. Lo que es una buena señal para el último día que se termina un curso:

"He aprendido bastante, espero volver a hacer cursos de este tipo".

"Ha sido una experiencia muy buena que no me importaría repetir".

"Ha sido la primera vez que he realizado un curso on-line, y desde luego no va a ser el último". 


\section{REFERENCIAS BIBLIOGRÁFICAS}

Pérez Garcías, A. (2002). Nuevas estrategias didácticas en entornos digitales para la enseñanza superior, en: Salinas, J.; Batista, A. (Coord.) Didáctica y Tecnología Educativa para una universidad en un mundo digital. Universidad de Panamá: Imprenta universitaria.

Prendes, M. P. (2003a). Aprendemos... ¿Cooperando o colaborando? Las claves del método, en: Martínez Sánchez, F. (Compilador)Redesdecomunicaciónen la enseñanza. Las nuevas perspectivas del trabajo corporativo. Barcelona: Paidós (95-127).

Prendes, M. P. (2003b). Trabajo colaborativo en espacios virtuales, en: Cabero, J.; Martínez, F. y Salinas,
J. (Coord.) Medios y herramientas de comunicación para la educación universitaria. Ciudad de Panamá: EDUTEC (193-206).

Sancho, J. M. (2003). En busca de respuestas para las necesidades educativas de la sociedad actual. Una perspectiva transdisciplinar de la Tecnología. Revista electrónica Fuentes, $n^{\circ} 4$ [en línea] Disponible en: http://www. revistafuentes.org/htm/portada. php?id volumen $=4$ [consulta 2007, 1 de mayo]

Salinas, J. (2003). Comunidades virtuales y aprendizaje digital, en: CD-ROM Edutec 2003. Caracas: Universidad Central de Venezuela.

\section{PERFIL ACADÉMICO Y PROFESIONAL DE LOS AUTORES}

Francisco Pavón Rabasco, profesor titular de la Universidad de Cádiz en el Área de didáctica y Organización Escolar, es docente de la asignatura Nuevas Tecnologías aplicadas a la Educación e investigador en temáticas como: Educación de Personas Mayores y Nuevas Tecnologías, las TIC's en las metodologías de trabajo propias del crédito europeo y los profesores de enseñanza no universitaria pioneros en el uso de las TIC's en los centros docentes.

E-mail: francisco.pavon@uca.es

DIRECCIÓN DEL AUTOR

Francisco Pavón Rabasco

C/Panamá 8

11100 San Fernando (Cádiz)

Juan Casanova Correa. Coordinador de la Titulación de Maestros Especialista en Audición y Lenguaje desde el curso académico 1995-96 hasta la fecha, en la E.U. de Magisterio 'Virgen de Europa' (Adscrita a la Universidad de Cádiz- España). Coordinador de la Titulación de Maestro Especialista en Primaria desde el curso 
académico 2003-04 hasta la fecha, en la E.U. de Magisterio 'Virgen de Europa' (Adscrita a la Universidad de Cádiz- España). Jefe del área departamental de Psicología, Medicina y Enfermería Coordinador durante los cursos académicos 98/99, 99/00, 0o/01. Profesor Colaborador en el Departamento de Didáctica de la Universidad de Cádiz desde julio de 2007. Profesor Titular en la Escuela Universitaria de Magisterio 'Virgen de Europa' (Adscrita a la UCA), desde 1995 hasta junio de 2007.

E-mail: juan.casanova@uca.es

DIRECCIÓN DEL AUTOR

Juan Casanova Correa

C/ Jaen, $\mathrm{n}^{\circ} 18$

11350. Castellar de la Frontera (Cádiz)

Fecha de recepción del artículo: 11/06/07

Fecha de aceptación del artículo: 17/09/07 
